\title{
Prevalence of a postoperative troponin leak in patients with cardiac risk factors undergoing knee and hip arthroplasty in a South African population
}

\author{
R D van Zyl, MB ChB, MMed (Orth), FC Orth (SA); M C Burger, BSc, BMedSc Hons, MMedSc, PhD; \\ J D Jordaan, MB ChB, FC Orth (SA), MMed (Orth)
}

Division of Orthopaedic Surgery, Department of Surgical Sciences, Faculty of Medicine and Health Sciences, Stellenbosch University and Tygerberg Hospital, Cape Town, South Africa

Corresponding author: $R$ D van Zyl (rdvanzyl@gmail.com)

\begin{abstract}
Background. Patients undergoing arthroplasty may have comorbidities that put them at risk of myocardial injury after non-cardiac surgery (MINS). MINS, a new clinical concept that has a different pathophysiology from conventional myocardial infarction, is related to a supplydemand mismatch ischaemia in the perioperative setting. MINS is often a silent event, and the diagnosis relies on cardiac biomarker testing such as troponin T. The incidence is estimated at $40 \%$, with a fourfold increase in morbidity and mortality risk 1 year post surgery.

Objectives. To determine the prevalence of postoperative troponin leak in a single-centre arthroplasty unit in patients with various cardiac risk factors undergoing hip or knee arthroplasty and investigate the differences in troponin $\mathrm{T}$ levels between comorbidities and different types of arthroplasty, i.e. total hip replacement (THR), total knee replacement (TKR) and neck of femur (NoF) fracture hip replacement.

Methods. A prospective, cross-sectional study of patients with one or more cardiac risk factors undergoing replacement surgery was conducted from October 2017 to April 2018. Troponin levels of all included patients were recorded on days 1 and 3 post surgery using a high-sensitivity cardiac troponin T assay (Roche hs-cTnT). A level of $>15 \mathrm{ng} / \mathrm{L}$ is considered abnormal and termed a positive troponin leak, while $>100 \mathrm{ng} / \mathrm{L}$ is considered suspected acute coronary syndrome (ACS).

Results. One hundred and sixty patients ( $n=66 \mathrm{THR}, n=55$ NoF hip replacement, $n=39$ TKR) were included. Sixty-eight patients (42\%) had a positive troponin leak, and in 6 of these cases ACS was suspected. The highest prevalence of troponin leak was recorded in patients undergoing NoF hip replacement (62\%), followed by TKR (46\%) and then THR (24\%). Sixty-two patients (38\%) had positive troponin levels on day 1 and 53 patients (33\%) had positive levels on day 3. Important patient cardiac risk factors were identified in the presence of a positive troponin leak, with ischaemic heart disease, hypertension, diabetes, renal disease, age $>65$ years and atrial fibrillation being statistically most likely.

Conclusions. Postoperative troponin surveillance is an inexpensive and reliable way to identify patients at risk of MINS and subsequently enhance early detection, medical optimisation and referral strategies. Simple interventions may improve outcomes and contribute to lower ACS rates and the timeous prevention of other complications. The prevalence of MINS in orthopaedic-specific patients in South Africa (SA) and other resource-constrained developing countries is unknown. Our finding of $42 \%$ positive troponin leaks raises awareness of this issue, and we recommend routine postoperative troponin surveillance for all arthroplasty units in SA.
\end{abstract}

S Afr Med J 2020;110(4):320-326. https://doi.org/10.7196/SAMJ.2020.v110i4.14133

Hip and knee arthroplasty are two of the most common operations performed worldwide. ${ }^{[1]}$ In the past decade, the occurrence of these surgical procedures has increased dramatically in step with the expansion and ageing of the world population. Technological advances and improvements in prosthetic design have also resulted in more indications and increased use. ${ }^{[2]}$ Hip replacement surgery has increased by $30 \%$ over the past two decades, while it is estimated that the demand for knee replacement surgery will increase fourfold by $2030 .^{[3,4]}$ Neck of femur (NoF) fractures are common emergency conditions in the geriatric population, and despite declines in incidence rates in certain populations, the worldwide number is also expected to increase fourfold by $2050 .{ }^{[5]}$ With reported 1-year mortality ranging between $12 \%$ and $37 \%$, this is the most expensive fracture to treat, as compared with other fractures, more patients die during hospitalisation, or within 1 month of sustaining the injury. ${ }^{[5,6]}$

Arthroplasty patients comprise a large proportion of individuals at risk of cardiac complications, as many of these patients are of advanced age, have one or more other cardiac risk factors, and could additionally suffer from the physiological stress associated with an
NoF fracture. ${ }^{[7,8]}$ Risk factors include uncontrolled hypertension, ischaemic heart disease (IHD), diabetes, renal disease, advanced age ( $>60$ years), heart failure, arrhythmias such as atrial fibrillation (AF), smoking, obesity, high cholesterol and poor nutritional status. ${ }^{[0,9-11]}$

Total hip and knee arthroplasty are classified as major surgeries that carry an increased risk of cardiac events perioperatively. ${ }^{[12]}$ In 2015 , Smith et al.$^{[1]}$ reported that the most common cause of death (affecting 7 out of 10 patients) following NoF fracture was myocardial infarction (MI). ${ }^{[1]}$

Myocardial injury after non-cardiac surgery (MINS) is a silent event that occurs within 30 days after non-cardiac surgery. ${ }^{[13,14]}$ It is thought to be a stress-induced ischaemia resulting from a myocardial oxygen supply and demand mismatch. ${ }^{[15,16]}$ Unfortunately up to $80 \%$ of these MIs are underdiagnosed, ${ }^{[17,18]}$ as the classic symptoms such as chest pain may be atypical or even absent. ${ }^{[19]}$ Because the mortality is reported to be similar to conventional MIs, ${ }^{[20-22]}$ an aggressive diagnostic and therapeutic approach is needed.

The diagnosis of MINS relies heavily on cardiac biomarkers ${ }^{[15]}$ such as a new high-sensitivity troponin $\mathrm{T}$ assay (hs-cTnT), which is the 
preferred biomarker in the diagnosis of MI, ${ }^{[23,24]}$ as well as exclusion of known non-ischaemic causes of perioperative troponin elevation such as sepsis and burns. ${ }^{[16,24,25]}$

Perioperative troponin surveillance is a simple and cost-effective method ${ }^{[26]}$ and of vital importance in diagnosing MINS. ${ }^{[13,27]}$ The test also provides important prognostic information ${ }^{[1,28]}$ when identifying patients at risk of a fatal outcome. ${ }^{[27-30]}$

Preventive strategies, medical optimisation and simple interventions, such as aspirin and statins, may improve outcome and survival. ${ }^{[13,17,31]}$ It is important to note, however, that none of these strategies has focused solely on the orthopaedic population. ${ }^{[19]}$ Under-appreciation of the extent of MINS, especially in elderly and cardiac patients undergoing arthroplasty, is therefore evident.

There are currently limited published data on the prevalence of MINS in orthopaedic-specific patients in South Africa (SA), and perioperative measurement of troponin levels is not considered standard protocol for patients undergoing arthroplasty. ${ }^{[16,32]}$ In addition, there is currently insufficient information to successfully compare patient comorbidities in different arthroplasty procedures.

We hypothesise that there may be an underdiagnosis of MINS, specifically in the domain of arthroplasty, and establishing a statistical significance in the prevalence rate could raise awareness and, along with the provision of early optimal medical intervention, reduce morbidity and mortality. ${ }^{[1,33]}$

\section{Objectives}

The primary objective was to determine the prevalence of postoperative troponin leak in a single-centre arthroplasty unit in patients with different cardiac risk factors undergoing hip or knee arthroplasty. A secondary objective was to investigate the differences in troponin $\mathrm{T}$ levels between various comorbidities and different types of arthroplasty, i.e. total hip replacement (THR), total knee replacement (TKR) and NoF hip replacement.

\section{Methods \\ Study design}

A prospective observational study was conducted including all patients undergoing knee and hip arthroplasty at Tygerberg Hospital, a tertiary academic institution in Cape Town, SA. Ethical approval (ref no. S17/02/042) and hospital clearance for the study were granted by the health research ethics committees of Stellenbosch University and Tygerberg Hospital, respectively. The study was registered with the National Health Research Database (ref. no. WC_201803_017).

\section{Participants}

Potential participants for the study were recruited over a period of 6 months from October 2017 to April 2018.

All patients admitted to the arthroplasty unit for joint replacement surgery who met the inclusion criteria were invited to participate in the study. Table 1 shows that patients eligible for the study: $(i)$ were undergoing either primary elective knee or hip arthroplasty or nonelective emergency arthroplasty for NoF fractures; (ii) were aged $\geq 40$ years or older; (iii) had one or more known cardiac risk factors (including but not limited to hypertension, IHD, smoking, diabetes, renal disease, obesity, high cholesterol, poor diet, excessive alcohol consumption and/or age $\geq 65$ years); and (iv) had received either regional and/or general anaesthesia. ${ }^{[9,10,16,19]}$ Patients who presented with sepsis and those with incomplete data on cardiac risk factors were excluded from the study (Table 1).

\section{Study procedures}

Patients were admitted as per the normal standard hospital protocol.

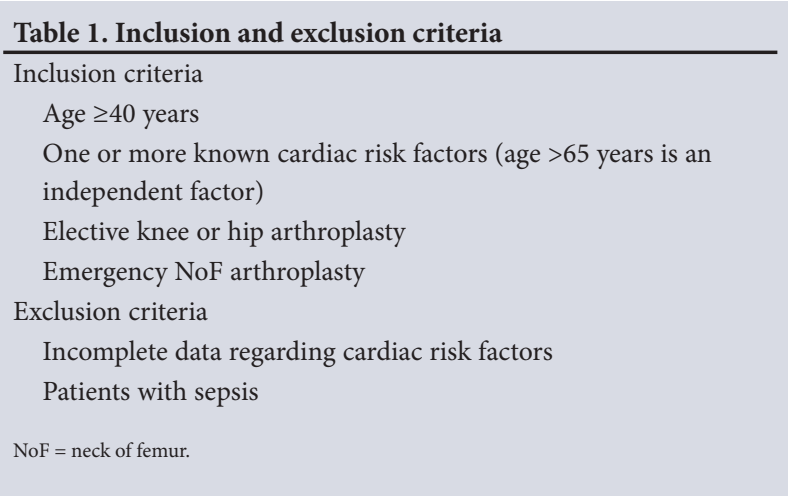

General characteristics (including age, presence of comorbidities and potential cardiac risk factors) were recorded from history and hospital charts. Five of these risk factors are referred to as the 'big five' because of their known association with a high risk of myocardial injury. ${ }^{[5,6,9-11,34]}$ These five risk factors are IHD, diabetes, renal disease, age $>65$ years and AF.

Written informed consent was obtained on admission and patients were also provided with a patient information leaflet. If the patient was unable to understand the process, a next of kin or legal guardian signed the consent on their behalf. An interpreter assisted with translation if needed.

A single blood sample $(2 \mathrm{~mL})$ was collected $(i)$ on day 1 (at least 12 hours postoperatively) and (ii) on day 3 postoperatively. These samples were then used to measure the serum troponin $\mathrm{T}$ levels of all included patients. This was done using a Roche 4th-generation Elecsys hs-cTnT assay. All results were recorded by the principal investigator. All test procedures were performed while the patients were still in hospital. Patients enrolled between 12 and 24 hours after surgery had blood samples drawn immediately once consent had been obtained, and testing continued as per the study protocol.

An hs-cTnT level of $>15 \mathrm{ng} / \mathrm{L}$ was considered abnormal and termed a positive troponin leak, ${ }^{[20,24,34]}$ while a cut-off level of $>100 \mathrm{ng} / \mathrm{L}$, a $50 \%$ change in an initial value of $<53 \mathrm{ng} / \mathrm{L}$, or a $20 \%$ change in an initial value between 53 and $100 \mathrm{ng} / \mathrm{L}$ were considered suspected acute coronary syndrome (ACS). ${ }^{[20,24,34,35]}$ For the purposes of this study, ACS was clinically diagnosed by an increase in the referenced hs-cTnT values above the reference range accompanied by ischaemic symptoms, ischaemic electrocardiogram (ECG) changes or new pathological Q-waves. ${ }^{[36]}$

Patients continued to receive standard care from the arthroplasty unit, which included additional troponin tests and/or ECGs, if warranted. All patients were reviewed daily for symptoms of myocardial ischaemia such as chest pain, dyspnoea, nausea, pallor, discomfort in other areas of the body (abdomen, shoulder, jaw), altered neurology and hypotension. ${ }^{[37]}$ If a patient met the requirements of a suspected ACS, they were managed and referred accordingly, as per the standard hospital protocol.

\section{Statistical analysis}

Data were analysed using STATISTICA version 13.2 (Statsoft Inc., USA). Data were tested for normality using the Kolmogorov-Smirnov test, and as the data were not normally distributed, data were expressed as medians and interquartile ranges (IQRs) or as numbers and percentages. Categorical data were expressed as frequencies and counts. Prevalence was determined as follows:

$$
\text { Prevalence }=\frac{\text { Number of patients with a positive test }}{\text { Total number of patients }}
$$


The effect of comorbidities on the risk of having a positive troponin $\mathrm{T}$ test was determined using Fisher's exact test. A $p$-value $<0.05$ was considered significant.

\section{Results \\ General characteristics}

A total of 170 patients were screened for potential inclusion in the study, and 160 were included (Fig. 1). The mean (standard deviation) age of the included participants was 66 (12) years, with $40 \%(n=64)$ male and $60 \%(n=96)$ female. Three individuals declined to participate in providing complete data for the study, and 7 did not meet the inclusion criteria.

A total of 66 patients $(42 \%)$ underwent elective THR, 55 patients (34\%) underwent NoF hip replacement, and 39 patients (24\%) underwent elective TKR (Fig. 1).

The median (interquartile range (IQR)) time to surgery was $2(1-4)$ days and the median time to discharge 4 ( 3 - 5.5) days. NoF patients waited slightly longer for surgery, with a median of $3(2-6)$ days, and were discharged after a median of $4(4-6)$ days (Table 2, Fig. 2).

\section{Clinical characteristics}

The overall prevalence of troponin leak was $42 \%(68 / 160)$ (Table 3), and 6 patients (4\%) were identified with a suspected ACS according to the diagnostic criteria. Similar patterns were observed when the prevalence was stratified for sex (supplementary Table 1, available at http://www.samj.org.za/public/ sup/14133.docx).

A positive troponin leak was detected in $39 \%$ of patients on day 1 following surgery, while $33 \%$ of patients tested positive on day 3 (Table 4). Not all patients who had tested positive on the first day $(n=62)$ tested positive again on day $3(n=14)$. Some of the patients who tested positive on day 3 $(n=53)$ had not tested positive on the first day $(n=5)$. Similar patterns were observed when prevalence was stratified for sex (supplementary Table 2, http://www.samj. org.za/public/sup/14133.docx).

Several cardiac risk factors and/or comorbidities were observed (Table 5). Patients identified with the 'big five' comorbidities IHD (odds ratio (OR) 4.7; 95\% confidence interval (CI) 1.9 - 11.4; $p<0.001$ ), renal disease (OR 17.6; 95\% CI $2.2-139.8 ; p<0.001)$, age $>65$ years (OR 6.3; 95\% CI $3.0-13.0 ; p<0.001$ ) and AF (OR 13.9; 95\% CI $1.7-112.5 ; p=0.002$ ) were more likely to have a positive troponin test than those without these comorbidities. Other cardiac risk factors of interest included

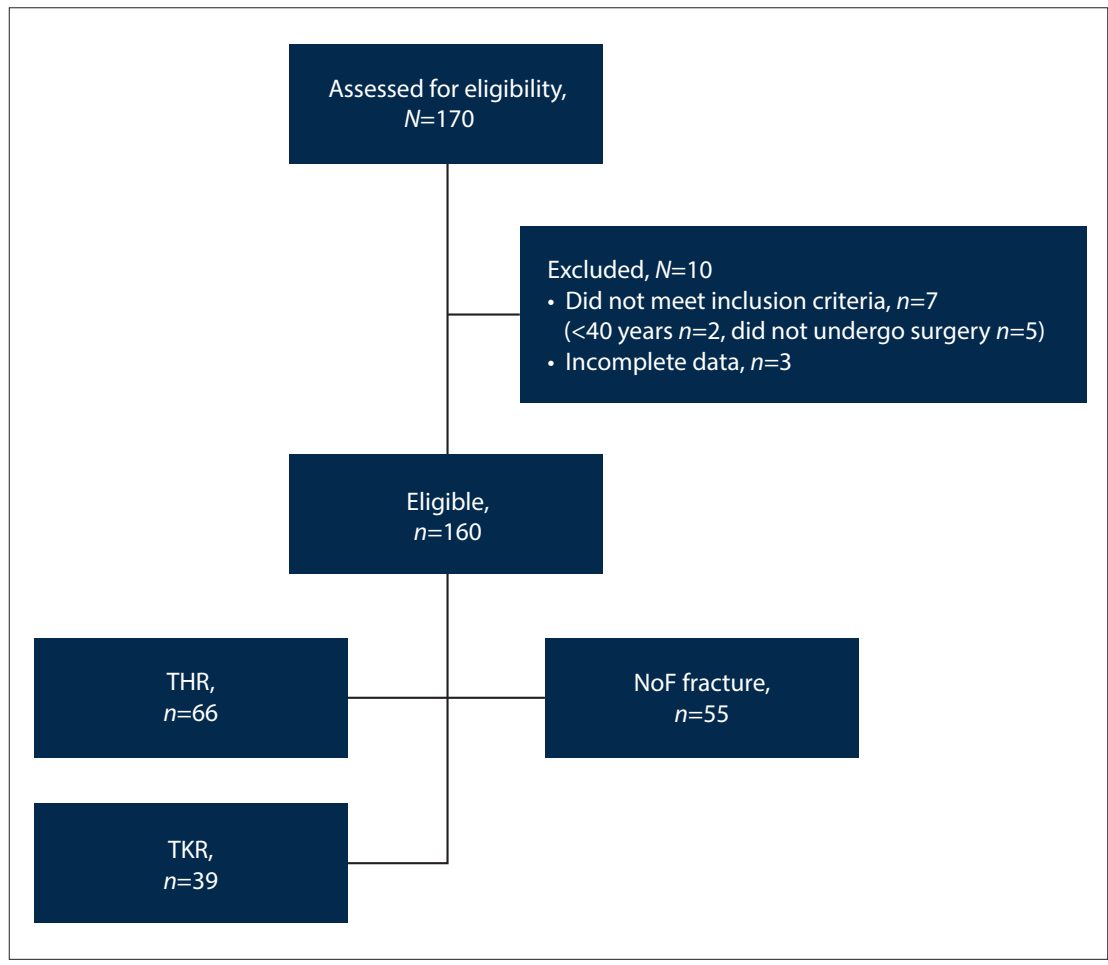

Fig. 1. Flowchart depicting patients enrolled in the study. $(T H R=$ total hip replacement; TKR $=$ total knee replacement; $\mathrm{NoF}=$ neck of femur.)

Table 2. Summary of time to surgery and time to discharge

\begin{tabular}{lll}
\multicolumn{3}{l}{ Table 2. Summary of time to surgery and time to discharge } \\
\hline All participants & Days to surgery, median (IQR) & Days to discharge, median (IQR) \\
NoF & $(1-4)$ & $4(3-5.5)$ \\
THR & $3(2-6)$ & $4(4-6)$ \\
TKR & $1(1-2)$ & $3(3-4)$ \\
& $1(1-2)$ & $4(3-6)$
\end{tabular}

$\mathrm{IQR}=$ interquartile range; $\mathrm{NoF}=$ neck of femur fracture; $\mathrm{THR}=$ total hip replacement; $\mathrm{TKR}=$ total knee replacement.

Table 3. Overview of the prevalence of a troponin leak between different arthroplasty procedures

\begin{tabular}{lll}
\hline & Positive leak, $\boldsymbol{n}(\%)$ & Negative leak, $\boldsymbol{n}(\%)$ \\
\hline All participants $(N=160)$ & $68(42.5)$ & $92(57.5)$ \\
NoF $(N=55)$ & $34(61.8)$ & $21(38.2)$ \\
THR $(N=66)$ & $16(24.2)$ & $50(75.8)$ \\
TKR $(N=39)$ & $18(46.2)$ & $21(53.8)$ \\
NoF = neck of femur fracture; THR = total hip replacement; TKR = total knee replacement.
\end{tabular}

Table 4. Overview of the prevalence of a troponin leak at day 1 and at day 3 post surgery

\begin{tabular}{lll}
\hline & Positive leak, $\boldsymbol{n}(\%)$ & Negative leak, $\boldsymbol{n}(\%)$ \\
\hline Day 1 & $62(38.8)$ & $98(61.3)$ \\
Day 3 & $53(33.1)$ & $107(66.9)$
\end{tabular}

patients with hypertension, who showed an increased risk of a positive troponin test compared with patients who did not have hypertension (OR 23.3; 95\% CI 1.5 - 4 332.5; $p<0.001$ ) (Table 5).

\section{Discussion}

\section{Primary outcomes}

The main finding of this study was that there was a $42.5 \%$ prevalence of postoperative troponin leak in patients with various 


\begin{tabular}{|c|c|c|c|c|}
\hline Comorbidity & Prevalence (total $N=160), n$ & Positive leak, $\boldsymbol{n}(\%)$ & OR $(95 \% \mathrm{CI})$ & $p$-value \\
\hline \multicolumn{5}{|l|}{ 'Big five’ } \\
\hline IHD & 29 & $21(72.4)$ & $4.7(1.9-11.4)$ & $<0.001$ \\
\hline Diabetes & 21 & $11(52.4)$ & $1.6(0.63-3.97)$ & 0.352 \\
\hline Renal disease & 12 & $11(91.7)$ & $17.6(2.2-139.8)$ & $<0.001$ \\
\hline Age $>65$ years & 89 & $54(60.7)$ & $6.3(3.0-13.0)$ & $<0.001$ \\
\hline $\mathrm{AF}$ & 10 & $9(90.0)$ & $13.9(1.71-112.5)$ & 0.002 \\
\hline \multicolumn{5}{|l|}{ Other } \\
\hline Hypertension & 147 & $68(46.3)$ & $23.3(1.5-4332.5)$ & $<0.001$ \\
\hline COPD & 34 & $11(32.4)$ & $0.6(0.26-1.28)$ & 0.242 \\
\hline Obesity & 18 & $6(32.4)$ & $0.5(0.3-1.3)$ & 0.334 \\
\hline Smoking & 49 & $13(26.5)$ & $0.4(0.2-0.9)$ & 0.023 \\
\hline Hypercholesterolaemia & 54 & $27(50.0)$ & $1.4(0.7-2.8)$ & 0.317 \\
\hline Poor nutritional status & 12 & $5(41.7)$ & $1.1(0.3-3.9)$ & 1.000 \\
\hline
\end{tabular}

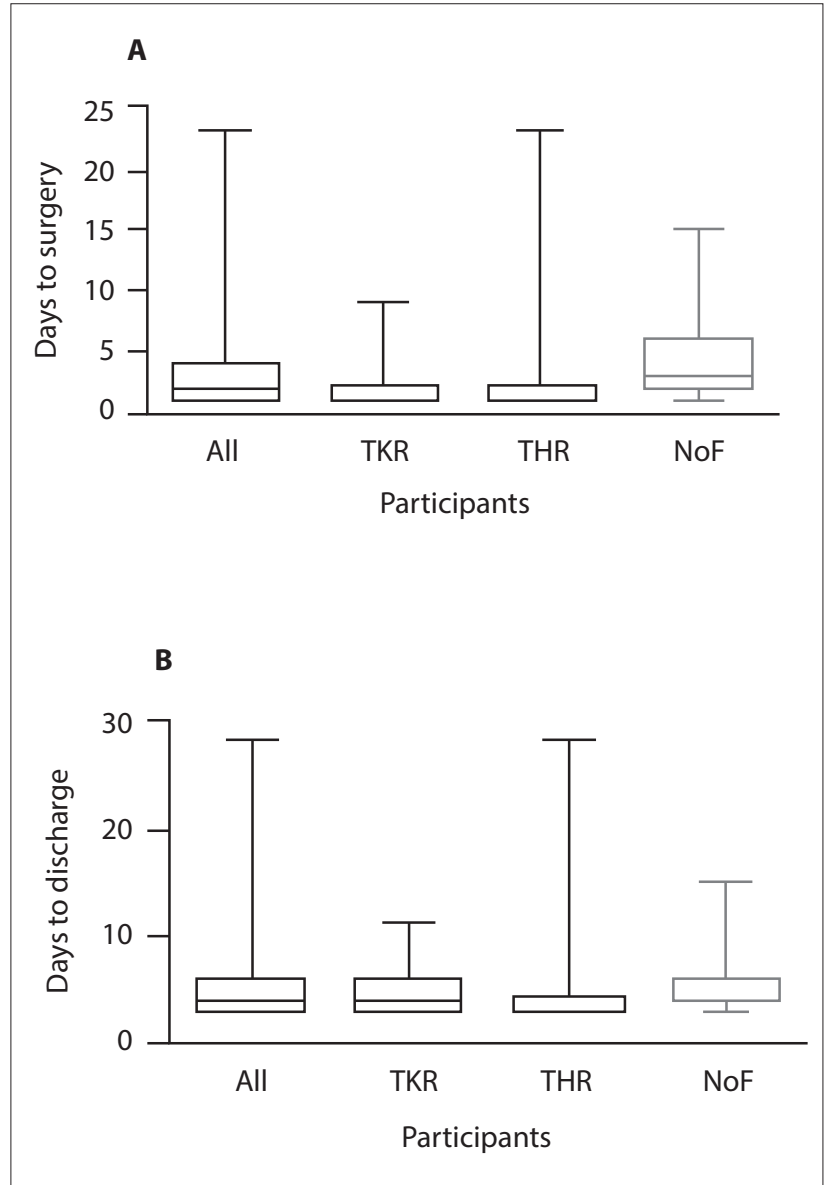

Fig. 2. Summary of time delays to surgery (A) and time to discharge (B) for all participants and for each surgical group. Data are presented as medians with interquartile and full ranges. $(T K R=$ total knee replacement; $T H R=$ total hip replacement; NoF $=$ neck of femur fracture.)

cardiac risk factors undergoing knee and hip arthroplasty. The need for arthroplasty procedures is on the increase in an ageing population. However, it is important to keep in mind that many patients who undergo these surgeries suffer from multiple comorbidities and are therefore susceptible to perioperative cardiovascular complications. ${ }^{[9,10,16,19,22]}$ Previous studies with varying numbers of participants have reported similar prevalence rates of troponin elevation. ${ }^{[20,25,38]}$
Dawson-Bowling et al. ${ }^{[38]}$ in the UK investigated patients aged $\geq 65$ years who presented with an NoF fracture over a 4-month period. They reported that of the cohort of 129 patients, 39\% showed a perioperative troponin rise. ${ }^{[38]}$ Global data suggest that $8 \%$ of adults aged $>45$ years suffer from MINS, and SA studies yield similar event rates. ${ }^{[1,26]}$ The local incidence of MINS in all orthopaedic patients is reported to be $10 \%$, with an in-hospital mortality rate of $7.7 \% .{ }^{[39-41]}$

When different types of arthroplasty procedures are compared, a higher prevalence of postoperative troponin leak is observed in patients with NoF fractures (67\%) than in those admitted for elective surgery, including THR (24\%) and TKR (46\%). Previous studies on major non-cardiac surgery have not specifically focused on orthopaedic and/or arthroplasty patients, and the outcomes of patients undergoing different types of arthroplasty, whether elective or emergency surgery, have also not been compared. ${ }^{[7,17,25]}$ In agreement with the findings presented in this study, NoF patients have previously been earmarked for special attention because they present with a higher incidence of postoperative troponin elevation (22-52\%) than the elective population $(0-9 \%)$. These patients are therefore a high-risk group in terms of MINS. ${ }^{[19]}$

The number of NoF fractures is expected to rise considerably by $2050,{ }^{[5]}$ with a high mortality at 1 year. ${ }^{[19]}$ Many of these patients die in hospital, or within 1 month after surgery. ${ }^{[5,6]}$ In 2015 , Smith et al. ${ }^{[1]}$ reported that the most common cause of death following NoF fracture is MI, affecting 7 out of 10 patients. It is therefore important that potential MIs be detected early. This increased risk is further emphasised by the high prevalence of postoperative troponin leaks in NoF patients.

A further interesting observation from the present study was the time to surgery and time to discharge for each procedure. Although time to discharge was similar for the various procedures, on average NoF patients waited longer for surgery than individuals scheduled for elective surgery. As time to surgery is a critical factor in the outcome of an NoF fracture, we postulate that the increased waiting time for surgery may have contributed to the higher prevalence of postoperative troponin leaks. ${ }^{[42]}$

It has been reported that surgery within $24-48$ hours leads to a reduction in 1-year mortality, ${ }^{[19,38]}$ and it is therefore recommended that unnecessary preoperative investigations should be avoided. In the UK, $>97 \%$ of patients who sustain an NoF fracture are operated on within 72 hours after injury. ${ }^{[42]}$

In a public health facility like our institution, reasons for delayed surgery are often multifactorial and include, but are not limited 


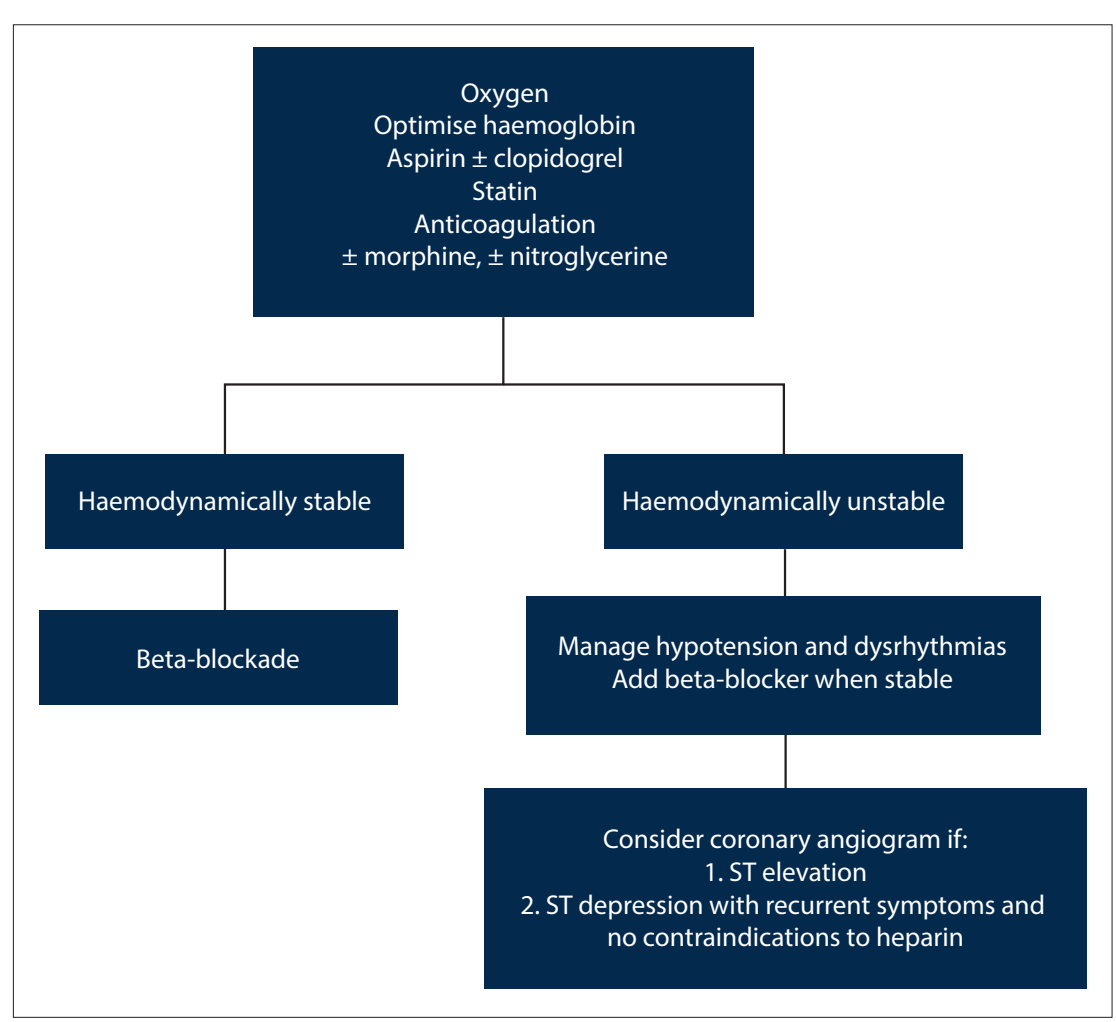

Fig. 3. Proposed algorithm for the management of patients with myocardial injury following noncardiac surgery ${ }^{[48]}$ (permission for use obtained from the author and from the publisher).

to, the following: (i) patient factors, such as uncontrolled medical comorbidities that may take a longer time to optimise prior to surgery; (ii) staff factors, including availability of trained surgical, anaesthetic and nursing staff; (iii) logistical factors, including available funding, theatre time, equipment and beds for postoperative highcare monitoring; and (iv) the overwhelming number of patients with NoF fractures requiring surgery presenting to the facility. ${ }^{[43]}$

Six patients (4\%) with a positive troponin elevation met the diagnostic criteria of a suspected ACS. These patients were generally older and had more than one cardiac risk factor. One patient required urgent resuscitation and was admitted to intensive care after an acute MI. We hypothesise that standard postoperative measures, which had already been initiated according to hospital protocol, in addition to early intervention could have contributed to an overall lower rate of progression to ACS in individuals identified with a troponin leak.

\section{Secondary outcomes}

Total hip and knee arthroplasty are classified as major surgeries and are known to be associated with a high risk of perioperative cardiac events. ${ }^{[12]}$ In addition, many patients undergoing arthroplasty have multiple comorbidities and are therefore at increased risk of MINS. ${ }^{[8,10]}$ There are also various surgical and anaesthetic perioperative factors associated with poor outcomes following hip and knee arthroplasty. ${ }^{[10]}$

Several cardiac comorbidities were identified, among them the 'big five'. Previous studies identified these comorbidities as independent indicators of a fatal outcome following hip or knee arthroplasty. ${ }^{[5,9,10,16,34]}$ Specifically, a history of IHD, renal disease, age $>65$ years, a history of $\mathrm{AF}$ and hypertension can increase the risk of troponin leak between 5- and 23-fold. Although the sample size of some of these comorbidities was small, and the results should therefore be interpreted with caution, being aware of these factors prior to surgery can help identify patients at risk. Surgical planning and the optimising of modifiable risk factors can ultimately prevent possible poor outcomes, especially in the case of elective surgery where there is sufficient time. ${ }^{[10,16,19]}$

It was interesting to note that diabetes was not identified as a risk factor for cardiac events in the present study, although the literature identifies it as such. ${ }^{[5,9,10,22]}$ A possible explanation is the small sample size of the subgroup of patients with diabetes and the fact that no information was recorded regarding control of the condition or whether or not insulin was being used. Similarly, smoking appeared to have a protective effect against troponin leak, a phenomenon highly contradictory to the literature. ${ }^{[9,10,44]}$ Once again, the small sample size may be partly to blame for this surprising finding.

Additionally, no background information on smoking behaviour was collected, such as history and number of cigarettes smoked per day. In both instances these results, which are highly contradictory to global literature, should therefore be interpreted with great care and assessed and evaluated in future studies.

Troponin is most likely to be elevated on the first day after surgery (38\%), and slightly less so on the third day (33\%). Although most events will be identified on the first day, it was important to note that 5 patients only tested positive on day 3 . These patients would have been missed if only a single test had been administered. Postoperative serum troponin levels demonstrate two peaks in elevation, the first at $18-24$ hours and the second 3 days after surgery. ${ }^{[19]}$ The VISION group demonstrated that troponin elevation during the first 3 days after noncardiac surgery was a major independent predictor of 30-day mortality. ${ }^{[17,25]}$ The study also reported a substantial decline in MINS events by day 3 . Although some events could statistically still present after this time, it is unlikely that any MINS events would have been missed. ${ }^{[25]}$ Guidelines recommend the daily measuring of troponins for $48-72$ hours after surgery in high-risk patients. ${ }^{[32]}$

It is also important to note that while most patients develop an elevated troponin level within the first 24 hours after surgery, early MI could also result in a patient being more susceptible to a fatal outcome from nonvascular complications such as pneumonia, which usually only develop on average 6 days after surgery. ${ }^{[22,25]}$

Troponin surveillance must be executed proactively and not selectively, as we cannot reliably predict who will suffer a postoperative leak. ${ }^{[33]} \mathrm{Up}$ to $80 \%$ of postoperative MIs occur in patients with no more than one cardiac risk factor, and efforts to reduce incidence should therefore not be limited to high-risk patients with multiple comorbidities alone. ${ }^{[45]}$

\section{Global implications}

Postoperative troponin testing as standard practice is feasible and may help to improve the prognosis, risk stratification and management of patients. ${ }^{[4]}$ At ZAR150 (USD10) per sample, this test is an inexpensive way to detect myocardial ischaemia and determine which patients are at risk of serious mortality. ${ }^{[23]}$ 
Based on 2011 private healthcare costs in South Africa, Torborg ${ }^{[2]}$ conducted an economic analysis of perioperative troponin monitoring after non-cardiac surgery. The cost saving associated with routine troponin surveillance amounted to ZAR16 700 ( USD1 170) per event, and surveillance did not yield an unnecessary increase in expenditure associated with subsequent inappropriate coronary angiography ${ }^{[26]}$ This analysis was further comparable to another model, described by Lurati Buse et al. ${ }^{[47]}$ where the incremental cost of avoiding a missed MINS event was calculated at CAD1 632 (2015 Canadian dollars).

From 1966 to 1986, pulmonary embolism (PE) and deep-vein thrombosis (DVT) were reported as having baseline mortality of $7 \%$ when associated with hip fractures. ${ }^{[32]}$ The VISION study recorded a reduced mortality of $0.07 \%$ that can be attributed to DVT prophylaxis. ${ }^{[25,32]}$ In the cases of DVT and PE, the proposed management of MINS advocates prevention and early intervention.

Observational data reported that $50 \%$ of patients have a 24 -hour window period of sustained low troponin leak from ischaemic myocytes alone prior to cell death and further elevation. ${ }^{[16]}$ Even after peak troponin, the median time to death varies from 9 to 12 days. ${ }^{[4]}$ Intervention and medical optimisation can therefore be significantly improved during this window period.

Current data suggest that simple measures could improve outcomes, with attention given to an increase in postoperative monitoring, optimisation of myocardial oxygen balance via haemodynamic support, and correction of anaemia and electrolyte disturbances. ${ }^{[16]}$ Biccard $^{[33]}$ introduced and modified a proposed algorithm that simplifies the management of patients with MINS according to haemodynamic stability and indicates when further intervention is needed (Fig. 3).

On the basis of recommendations published in the South African Medical Journal ${ }^{[16]}$ and the South African Orthopaedic Journal, ${ }^{[32]}$ this algorithm has also been adopted in our hospital division.

A therapeutic decision based on hs-cTnT needs to be considered carefully, as therapy could be harmful and increase the risk of bleeding. ${ }^{[14]}$ The MANAGE study evaluated the impact of anticoagulation on perioperative vascular complications after MINS, and provisional results indicate improved outcomes. ${ }^{[48]}$

The POISE study ascertained that beta-blockers were useful in haemodynamically stable patients, but did not rule out the risk of stroke, hypotension, bradycardia and death. ${ }^{[45,49]}$ Troponin surveillance and the use of simple therapies, such as aspirin and a statin for 30 days, may improve outcomes, with a 25\% risk reduction in major cardiovascular complications. ${ }^{[26,48]}$

\section{Study limitations}

This study, although prospective, only included early postoperative prevalence and not preoperative troponin levels, which could be raised prior to surgery, especially in the NoF group. Preoperative testing could help to determine whether troponin elevation occurred before and/or after surgery and to quantify the extent of both. ${ }^{[15]}$ This study also did not include any medium- and/or long-term follow-up of patients who experienced positive troponin leak/s to determine future implications.

The recording of patient cardiac risk factors included conditions sourced from patient history. However, the chronicity and/or severity of each condition, how well it was being controlled, and/or additional information detailing treatment were not specified. ${ }^{[44]}$ Until further research has been done, all comorbidities should be managed on merit. In accordance with hospital protocol and in terms of Biccard's proposed algorithm, ${ }^{[50]}$ patients receive standard care. This study did not include discussion on the possible effects of an intervention or its contribution to the prevalence of a troponin leak, either on day 1 or day 3 after surgery.

\section{Recommendations}

Continued research into the pathophysiology of MINS is necessary, as data on its prevention and/or treatment are limited, especially in the areas of orthopaedic surgery and patients exhibiting various cardiac risk factors.

Troponin surveillance is a simple and inexpensive method that can be successfully employed in the recognition of MINS, allowing early medical optimisation of at-risk patients and prevention of serious complications. Future research should include long-term follow-up studies, perioperative comparisons, and additional implementations aimed at the prevention and/or management of MINS.

\section{Conclusions}

MINS, especially in conjunction with arthroplasty, is underdiagnosed and data on its prevalence in the SA population are limited. The reported $42 \%$ prevalence of postoperative troponin leak in our study population raises a new awareness regarding patients at risk of MINS, as well as the considerable health burden that it may pose. Additionally, the higher prevalence of postoperative troponin leak of $62 \%$ in NoF patients underscores the importance of troponin surveillance in this at-risk group.

We recommend that routine troponin surveillance be done in all arthroplasty units in SA in patients aged $>40$ years, those with one or more cardiac risk factors, and those who have sustained an NoF fracture.

\section{Declaration. None.}

Acknowledgements. The authors thank the medical personnel (doctors and nursing staff) of Tygerberg Hospital who assisted in taking blood samples for troponin testing.

Author contributions. RDvZ: contributed to the conception and design of the research, acquisition of data, drafting and reading of the manuscript; JDJ: contributed to the conception and design of the research, drafting and reading of the manuscript; MCB: contributed to the conception and design of the research, drafting and reading of the manuscript.

Funding. None.

Conflicts of interest. None.

1. Smith EJ, Maru M, Siegmeth A. Thirty-day mortality after elective hip and knee arthroplasty. Surgeon 2015;13(1):5-8. https://doi.org/10.1016/..surge.2013.12.004

2. Learmonth ID, Young C, Rorabeck C. The operation of the century: Total hip replacement. Lancet 2007;370(9597):1508-1519. https://doi.org/10.1016/s0140-6736(07)60457-7

3. Pabinger C, Lothaller H, Geissler A. Utilization rates of knee-arthroplasty in OECD countries. Osteoarthr Cartil 2015;23(10):1664-1673. https://doi.org/10.1016/j.joca.2015.05.008

4. OECD. Hip and knee replacement. Health at a Glance 2017. Paris: OECD Publishing, 2017:178-179. https://doi.org/10.1787/19991312

5. Fisher A, Fisher L, Srikusalanukul W, Smith PN. Usefulness of simple biomarkers at admission as independent indicators and predictors of in-hospital mortality in older hip fracture patients. Injury 2018;49(4):829-840. https://doi.org/10.1016/j.injury.2018.03.005

6. Vallet $\mathrm{H}$, Breining A, Le Manach Y, et al. Isolated cardiac troponin rise does not modify the prognosis in elderly patients with hip fracture. Medicine (Baltimore) 2017;96(7):e6169. https://doi.org/10.1097/
md.0000000000006169

7. Le Manach Y, Collins G, Bhandari M, et al. Outcomes after hip fracture surgery compared with elective total hip replacement. JAMA 2015;314(11):1159-1166. https://doi.org/10.1001/jama.2015.10842

8. Jain NB, Guller U, Pietrobon R, Bond TK, Higgins LD. Comorbidities increase complication rates in patients having arthroplasty. Clin Orthop Relat Res 2005;435:232-238. https://doi.org/10.1097/01.
ing in patients having arthropl

9. Hoff J, Wehner W, Nambi V. Troponin in cardiovascular disease prevention: Updates and future direction. Curr Atheroscler Rep 2016;18(3):12. https://doi.org/10.1007/s11883-016-0566-5

10. Memtsoudis SG, Rosenberger P, Walz JM. Critical care issues in the patient after major joint replacement. J Intensive Care Med 2007;22(2):92-104. https://doi.org/10.1177/0885066606297692

11. Mueller-Hennessen M, Lindahl B, Giannitsis E, et al. Diagnostic and prognostic implications using age- and gender-specific cut-offs for high-sensitivity cardiac troponin $\mathrm{T}$ - sub-analysis from the TRAPID-AMI study. Int J Cardiol 2016;209:26-33. https://doi.org/10.1016/j.ijcard.2016.01.213

12. Bass AR, Rodriguez T, Hyun G, et al. Myocardial ischaemia after hip and knee arthroplasty: Incidence and risk factors. Int Orthop 2015;39(10):2011-2016. https://doi.org/10.1007/s00264-015-2853-0

13. Botto F, Alonso-Coello P, Chan MT, et al. Myocardial injury after noncardiac surgery. Anesthesiology 2014;120(3):564-578. https://doi.org/10.1097/aln.0000000000000113 
14. Chapalain $\mathrm{X}$, Huet O. Post-operative high sensitivity troponin $\mathrm{T}(\mathrm{hs} \mathrm{TnT} \mathrm{T})$ : Toward an extending use for diagnosis and management of myocardial injury after noncardiac surgery. J Thorac Dis 2017;9(8):2231diagnosis and management of myocardial injur
2234. https://doi.org/10.21037/itd.2017.06.146

15. Brown JC, Samaha E, Rao S, et al. High-sensitivity cardiac troponin $\mathrm{T}$ improves the diagnosis of perioperative MI. Anesth Analg 2017;125(5):1455-1462. https://doi.org/10.1213/ane.0000000000002240 16. Coetzee E, Biccard BM. Myocardial injury after non-cardiac surgery: Time to shed the ignorance. S Afr Med J 2018;108(6):464-467. https://doi.org/10.7196/SAMJ.2018.v108i6.13346

17. Khan J, Alonso-Coello P, Devereaux PJ. Myocardial injury after noncardiac surgery. Curr Opin Cardiol 2014;29(4):307-311. https://doi.org/10.1097/hco.0000000000000069

18. Devereaux PJ, Biccard BM, Sigamani A, et al. Association of postoperative high-sensitivity troponin levels with myocardial injury and 30-day mortality among patients undergoing noncardiac surgery. JAMA 2017;317(16):1642-1651. https://doi.org/10.1001/jama.2017.4360

19. Chong CP, van Gaal WJ, Savige J, Lim WK. Cardiac injury and troponin testing after orthopaedic surgery. Injury 2011;42(9):855-863. https://doi.org/10.1016/j.injury.2009.10.015

20. Chong CP, Lam QT, Ryan JE, Sinnappu RN, Lim WK. Incidence of post-operative troponin I rises and 1-year mortality after emergency orthopaedic surgery in older patients. Age Ageing 2008;38(2):168174. https://doi.org/10.1093/ageing/afn231

21. McCarthy CP, Vaduganathan M, Januzzi JL. Type 2 myocardial infarction - diagnosis, prognosis, and treatment. JAMA 2018;320(5):433-434. https://doi.org/10.1001/jama.2018.7125

22. Biccard BM, Devereaux PJ, Rodseth RN. Cardiac biomarkers in the prediction of risk in the noncardiac surgery setting. Anaesthesia 2014;69(5):484-493. https://doi.org/10.1111/anae.12635

23. Xu R-Y, Zhu X-F, Yang Y, Ye P. High-sensitive cardiac troponin T. J Geriatr Cardiol 2013;10(1):102-109.

24. Jardine RM, Dalby AJ, Klug EQ, et al. Consensus statement on the use of high sensitivity cardiac troponins. SA Heart 2012;9(3):210-215. http://www.sasci.co.za/uploads/files/SA_Heart_Consensus Statement_2012_Use_of_high_sensitivity_cardiac_troponins.pdf (accessed 20 February 2020).

25. Botto F, Alonso-Coello P, Chan MT, et al.; VISION Writing Group. Myocardial injury after noncardiac surgery: A large, international, prospective cohort study establishing diagnostic criteria noncacteristics, predictors, and 30-day outcomes. Anesthesiology 2014;120(3):564-578. https://doi.
char characteristics, predictors, and 30-
org/10.1097/ALN.0000000000000113

26. Torborg A, Ryan L, Kantor G, Biccard BM. The pharmacoeconomics of routine postoperative troponin surveillance to prevent and treat myocardial infarction after non-cardiac surgery. S Afr Med J 2014;104(9):619-623. https://doi.org/10.7196/SAMJ.7654

27. Talsnes $\mathrm{O}$, Hjelmstedt $\mathrm{F}$, Dahl $\mathrm{OE}$, Pripp AH, Reikerås $\mathrm{O}$. Clinical and biochemical prediction of early fatal outcome following hip fracture in the elderly. Int Orthop 2011;35(6):903-907. https://doi. org/10.1007/s00264-010-1149-7

28. Devereaux PJ, Goldman L, Yusuf S, et al. Surveillance and prevention of major perioperative ischemic cardiac events in patients undergoing noncardiac surgery: A review. CMAJ 2005;173(7):779-788. https://doi.org/10.1503/cmaj.050316

29. Lee TH, Thomas EJ, Ludwig LE, et al. Troponin $\mathrm{T}$ as a marker for myocardial ischemia in patient undergoing major noncardiac surgery. Am J Cardiol 1996;77(12):1031-1036. https://doi.org/10.1016/ s0002-9149(96)00126-9

30. Higham H, Sear JW, Sear YM, Kemp M, Hooper RJL, Foex P. Peri-operative troponin I concentration as a marker of long-term postoperative adverse cardiac outcomes - a study in high-risk surgical patients. Anaesthesia 2004;59(4):318-323. https://doi.org/10.1111/j.1365-2044.2004.03660.x

31. Sessler DI, Devereaux PJ. Perioperative troponin screening. Anesth Analg 2016;123(2):359-360. https://doi.org/10.1213/ane.0000000000001450

32. Simpson GC, Marais LC, Rodseth R. The silent killer: Myocardial injury after non-cardiac surgery (MINS). SA Orthop J 2018;17(3). https://doi.org/10.17159/2309-8309/2018/v17n2al

33. Biccard BM. Detection and management of perioperative myocardial ischemia. Curr Opin Anaesthesiol 2014;27(3):336-343, https://doi.org/10.1097/aco.0000000000000071
34. Duceppe E, Parlow J, MacDonald P, et al. Canadian Cardiovascular Society guidelines on perioperative cardiac risk assessment and management for patients who undergo noncardiac surgery. Can J Cardiol 2017;33(1):17-32. https://doi.org/10.1016/j.cica.2016.09.008

35. Roche Dignostics. Roche Troponin T-high sensitive. https://diagnostics.roche.com/global/en/products/

params/elecsys-troponin-t-high-sensitive-tnt-hs.htmll\#productInfo (accessed 4 March 2020).
36. Mendis S, Thygesen K, Kuulasmaa K, et al. World Health Organization definition of myocardial infarction: 2008 - 09 revision. Int J Epidemiol 2011;40(1):139-146. https://doi.org/10.1093/ije/dyq165

7. Aronow WS. Silent MI prevalence and prognosis in older patients diagnosed by routin electrocardiograms. Geriatrics 2003;58(1):24-40.

38. Dawson-Bowling S, Chettiar K, Cottam H, et al. Troponin Tas a predictive marker of morbidity in patient with fractured neck of femur. Injury 2008;39(7):775-780. https://doi.org/10.1016/j.injury.2008.01.025

39. Phaff M, Goga I, Biccard B. Myocardial injury after major orthopedic surgery and the increased risk in 30 day mortality. Presented at the 23rd Biennial Congress of the SA Arthroplasty Society, 2015. https:// www.researchgate.net/publication/275213030_Myocardial_injury_after_major_orthopedic_surgery_ and_increased_mortality (accessed 4 March 2020).

40. Duceppe E, Yusuf S, Tandon V, et al. Design of a randomized placebo-controlled trial to assess dabigatran and omeprazole in patients with myocardial injury after noncardiac surgery (MANAGE). Can J Cardiol 2018;34(3):295-302. https://doi.org/10.1016/j.cjca.2018.01.020

41. Urban MK, Jules-Elysee K, Loughlin C, Kelsey W, Flynn E. The one year incidence of postoperative myocardial infarction in an orthopedic population. HSS J 2008;4(1):76-80. https://doi.org/10.1007/ s11420-007-9070-3

42. Morrissey N, Iliopoulos E, Osmani AW, Newman K. Neck of femur fractures in the elderly: Does every hour to surgery count? Injury 2017;48(6):1155-1158. https://doi.org/10.1016/j.injury.2017.03.007

3. Conradie FC, Pienaar G, Jordaan K, Burger M. The timing of arthroplasty for femoral neck fractures. Presented at the 64th South African Orthopaedic Congress, 3 - 6 September 2018 (paper 108). SA Ortho J 2019;18(1):26. http://saoajournal.ps.studio/index.php/saoj/article/view/291 (accessed 4 March 2020).

44. US Department of Health and Human Services. How tobacco smoke causes disease: The biology and behavioural basis for smoking-attributable disease: A report of the Surgeon General. PsycEXTRA Dataset. American Psychological Association (APA), 2010.

45. Van Klei WA, Bryson GL, Yang H, Forster AJ. Effect of $\beta$-blocker prescription on the incidence of postoperative myocardial infarction after hip and knee arthroplasty. Anesthesiology 2009:111(4):717724. https://doi.org/10.1097/aln.0b013e3181b6a761

46. Van Waes JAR, Nathoe HM, de Graaff JC, et al. Myocardial injury after noncardiac surgery and its association with short-term mortality. Circulation 2013;127(23):2264-2271. https://doi.org/10.1016/j jvs.2013.10.047

47. Lurati Buse G, Manns B, Lamy A, et al. Troponin T monitoring to detect myocardial injury after noncardiac surgery: A cost-consequence analysis. Can J Surg 2018;61(3):185-194. https://doi org/10.1503/cjs.010217

48. Devereaux PJ. Management of Myocardial Injury After Noncardiac Surgery Trial (MANAGE). ClinicalTrials.gov, US National Library of Medicine, 2018. https://clinicaltrials.gov/show/NCT0166110 (accessed 4 March 2020).

49. Devereaux PJ, Yang H, Yusuf S, et al. Effects of extended-release metoprolol succinate in patients undergoing non-cardiac surgery (POISE trial): A randomised controlled trial. Lancet 2008;371(9627):1839-1847. https://doi.org/10.1016/s0140-6736(08)60601-7

50. Biccard BM. Peri-operative myocardial infarction. S Afr J Anaesth Analg 2010;16(1):44-46. https://doi. org/10.1080/22201173.2010.10872633

Accepted 10 September 2019. 